\title{
HOMEOMORPHISMS OF QUATERNION SPACE AND PROJECTIVE PLANES IN FOUR SPACE
}

\author{
T. M. PRICE \\ (Received 6 January 1975; revised 4 March 1975)
}

\begin{abstract}
It is known that all locally flat projective planes in $S^{4}$ have homeomorphic normal disk bundles. In this paper we investigate the homeomorphisms of $Q^{3}$ ( $\equiv$ boundary of the normal disk bundle) onto itself. We show that a homeomorphism of $Q^{3}$ onto itself is determined, up to isotopy, by the outerautomorphism of $\pi_{1}\left(Q^{3}\right)$ that it induces. Since $Q^{3}$ is an irreducible, not sufficiently large 3-manifold with finite fundamental group this characterization is interesting in its own right. The characterization of homeomorphisms is then used to study certain questions about embeddings of the projective plane in $S^{4}$. One result is that there are at most two distinct projective planes in $S^{4}$ with a given complement.
\end{abstract}

If $P$ and $P^{\prime}$ are smooth embeddings of the projective plane in $S^{4}$ then the normal bundle to $P$ is equivalent to the normal bundle to $P^{\prime}$ (see Theorem I of Massey (1974)). In fact, using smoothing theory, the above result can be extended to the case where $P$ and $P^{\prime}$ are $P L$ locally flat embeddings. Furthermore the normal disk bundle is constructed in Massey (1969) and in Price and Roseman (1975). In the latter paper it is shown that the boundary of this normal disk bundle, denoted $Q^{3}$, is a compact 3-manifold whose fundamental group is the quaternion group $\left\{a, b: a^{2}=b^{2}=(a b)^{2}\right\}$ and its universal cover is $S^{3}$. This 3-manifold, which we refer to as quaternion space, arises in a number of problems in topology (see page 198 of Seifert and Threlfall (1934) for an early reference). As mentioned above, it is the quotient space of $S^{3}$ modulo the action of the quaternion group on $S^{3}$. The referee has also pointed out that in Borel (1953), $Q^{3}$ is identified as $0(3) / Q(3)$. Using this fibration, as well as some related principal fibrations, Borel shows that $S_{3}$ (the symmetric group on 3 letters) acts on $Q^{3}$. In Corollary 1 to Theorem 1 of this paper we show that the group of isotopy classes of homeomorphisms of $Q^{3}$ onto itself is, in fact, isomorphic to $S_{3}$.

Having classified the homeomorphisms of $Q^{3}$ onto itself, we show (analogous to Gluck's results on embeddings of $S^{2}$ in $S^{4}$, see Gluck (1962)) 
that there exist at most two distinct embeddings of $P^{2}$ in $S^{4}$ with a given complement. We also point out that $Q^{3}$ is irreducible, not sufficiently large and has finite fundamental group. Hence the classification of homeomorphisms by their induced outerautomorphisms on $\pi_{1}\left(Q^{3}\right)$ (analogous to Waldhausen (1968)) is at least as interesting as the applications to embeddings of projective planes in $S^{4}$.

The notation used in the paper is quite standard. We write $\mathscr{H}(X)$ to denote the group of orientation preserving homeomorphisms of $X$ onto itself. We write $\mathscr{H}_{\text {id }}(X)$ to denote the subgroup of homeomorphisms isotopic to the identity. We write $\mathscr{I}(X)$ to denote the quotient group $\mathscr{H}(X) / \mathscr{H}_{\text {id }}(X)$ of isotopy classes of homeomorphisms of $X$ onto itself. If $G$ is a group, we write Aut $G$ to denote the automorphisms of $G$, Inaut $G$ to denote the innerautomorphisms of $G$ and Outaut $G$ to denote the quotient group Aut $G$ /Inaut $G$ of outerautomorphisms of $G$.

The normal disk bundle to a projective plane in $S^{4}$ is discussed in Price and Roseman (1975) but we give a brief description of it here. Let $K^{3}$ be the solid Klein bottle

$$
[0,1] \times B^{2} / 0 \times(x, y) \equiv 1 \times(-x, y)
$$

where $B^{2}$ denotes the unit disk in Euclidean 2-space with Cartesian coordinates. Let $F: S^{1} \times B^{2} \rightarrow K^{3}$ be defined by

$$
F(\theta \times(x, y))= \begin{cases}\frac{\theta}{\pi} \times(x, y) & 0 \leqq \theta \leqq \pi \\ \frac{\theta-\pi}{\pi} \times(-x, y) & \pi \leqq \theta \leqq 2 \pi .\end{cases}
$$

Let $M^{4}$ denote the mapping cylinder of $F$

$$
\text { (i.e., } M^{4}=\left(S^{1} \times B^{2}\right) \times[0,1] \underset{(\theta,(x, y), 0)=F(\theta,(x, y))}{\left.\bigcup^{3}\right) .} \text {. }
$$

Notice that $M^{2}=\left(S^{1} \times(0,0) \times[0,1] \cup F\left(S^{1} \times(0,0)\right)\right.$ is a Moebius band embedded in $M^{4}$. We complete the normal disk bundle by attaching $D^{2} \times B^{2}$ to $M^{4}$ by the map $\phi:\left(\partial D^{2}\right) \times B^{2} \rightarrow S^{1} \times B^{2} \times\{1\} \subseteq M^{4}$ given by $\phi(\alpha \times(x, y))=$ $\alpha \times \rho_{2 \alpha}(x, y) \times\{1\}$ where $\rho_{2 \alpha}$ is rotation of $B^{2}$ by an angle of $2 \alpha$ radians $\left(D^{2}\right.$ denotes a 2-disk). Thus $\nu=M^{4} \cup_{\phi} D^{2} \times B^{2}$. Note that $P^{2}=M^{2} \cup_{\phi} D^{2} \times(0,0)$ is a projective plane and $\nu$ is clearly a disk bundle over $P^{2}$. This is, in fact, the normal disk bundle structure. 
The boundary of $\nu$ is now easily described. Let $K^{2}$ denote the boundary of $K^{3}$ and let $T^{2}$ denote the boundary of $S^{1} \times B^{2}$. Let $M^{3} \subseteq \partial M^{4}$ denote the submapping cylinder

$$
\begin{aligned}
M^{3} & =\left(S^{1} \times \partial\left(B^{2}\right)\right) \times[0,1] \bigcup_{(\theta,(x, y), 0)=F(\theta,(x, y))} K^{2} \\
& =T^{2} \times[0,1] \bigcup_{F} K^{2} .
\end{aligned}
$$

Finally $\partial \nu$, which will usually be denoted, $Q^{3}$ is just $M^{3} \cup_{\phi} D^{2} \times\left(\partial B^{2}\right)$. We usually write $T^{3}$ for $D^{2} \times \partial B^{2}$. Figure 1 gives a useful schematic diagram of $Q^{3}$.

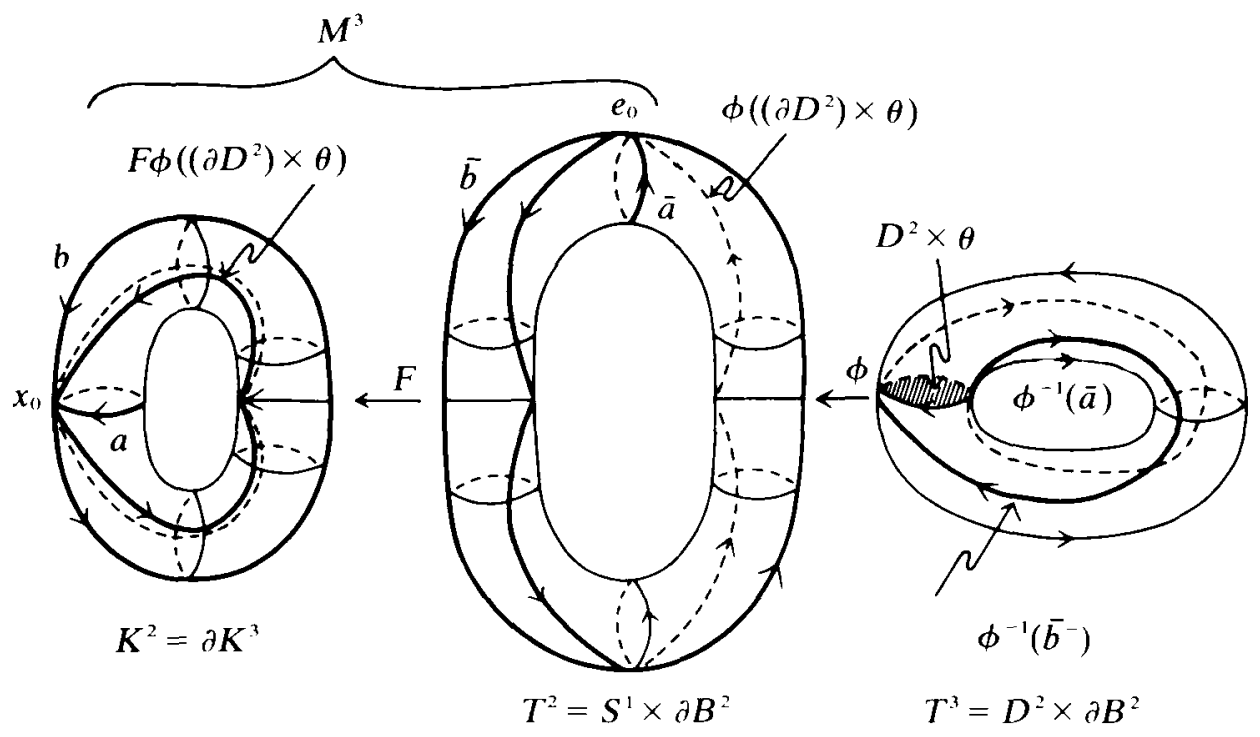

Figure 1

We will always mean the symbols $K^{2}, T^{2}, M^{3}$ and $T^{3}$ to denote the subsets of $Q^{3}$ described above. Also we will always use the symbols $a$ and $b$ to denote the oriented meridian and longitude on $K^{2}$ as shown in Figure 1 and the symbols $\bar{a}$ and $\bar{b}$ to denote the oriented "meridian" and "Iongitude" on $T^{2}$ as shown in Figure 1. Note that $F$ takes $\bar{a}$ homeomorphically onto $a$ and $F$ takes $\bar{b}$ by the 2 to 1 covering map onto $b$. We will use the points $x_{0}$ and $e_{0}$ as base points for fundamental groups. Using Van Kampen's theorem one easily computes $\pi_{1}\left(Q^{3}, x_{10}\right)$ to be the quaternion group (with eight elements) which can be presented $\left\{a, b: a^{2}=b^{2}=(a b)^{2}\right\}$ (we will use $a$ and $b$ to represent 
elements of $\pi_{1}\left(Q^{3}, x_{0}\right)$ as well as the meridian and longitude of $\left.K^{2}\right)$. The following list of elements and some equivalent words of $\pi_{1}\left(Q^{3}, x_{0}\right)$ is useful.

$$
\begin{array}{lll}
1=a^{4}=b^{4}=(a b)^{4} & b=a b a=a b^{-1} a^{-1}=a^{-1} b^{-1} a \\
a=b a b=b a^{-1} b^{-1}=b^{-1} a{ }^{1} b & b^{3}=b^{-1}=a b a^{-1}=a^{-1} b a \\
a^{2}=b^{2}=(a b)^{2}=(b a)^{2} & a b=(b a)^{-1}=b^{-1} a=b a^{-1} \\
a^{3}=a^{-1}=b a b^{-1}=b^{-1} a b & b a=(a b)^{-1}=a^{-1} b=a b^{-1} .
\end{array}
$$

It is easy to list the automorphisms and inner automorphisms of $\pi_{1}\left(Q^{3}, x_{0}\right)$. To get an automorphism, $\alpha$, let $\alpha(a)$ be anything except 1 or $b^{2}$. Then let $\alpha(b)$ be anything except $1, b^{2}, \alpha(a)$ or $\alpha(a)^{-1}$. This yields the 24 automorphisms (see page 74 of Passman (1968)). Conjugation by $a$ or $a^{-1}$ fixes $a$ and sends $b$ to $b^{-1}$. Conjugation by $b$ or $b^{-1}$ fixes $b$ and sends $a$ to $a^{-1}$. Conjugation by $a b$ or $b a$ sends $a$ to $a^{-1}$ and $b$ to $b^{-1}$. Conjugation by 1 or $a^{2}$ fixes everything since 1 and $a^{2}=b^{2}$ lie in the center.

Finally a couple of comments about the irreducibility of $M^{3}$ and $Q^{3}$ and the non-sufficiently largeness of $Q^{3}$. It is quite easy to construct the covering space of $Q^{3}$ corresponding to the subgroup $\left\{1, a, a^{2}, a^{3}\right\}$ of $\pi_{1}\left(Q^{3}, x_{0}\right)$ and identify it as the lens space $L(4,1)$. Hence the universal cover of $Q^{3}$ is $S^{3}$. Hence $Q^{3}$ is irreducible (each 2-sphere bounds a 3-cell). Since $\pi_{1}\left(T^{3}\right) \rightarrow \pi_{1}\left(Q^{3}\right)$ is not the zero homomorphism it follows that $T^{3}$ is not contained in a 3-cell in $Q^{3}$ hence $M^{3}$ is also irreducible. The projective plane is the only surface with a small enough fundamental group to embed incompressibly in $Q^{3}$ but its regular neighborhood would be a punctured projective 3-space whose boundary 2 -sphere does not bound a 3-cell. Hence $Q^{3}$ is not sufficiently large.

\section{Homeomorphisms on $Q^{3}$}

In this section we construct several homeomorphisms of $Q^{3}$. In the next section we will show that these homeomorphisms generate all of the isotopy classes of homeomorphisms of $Q^{3}$. In classifying the isotopy classes we will use the action of a homeomorphism on $\pi_{1}\left(Q^{3}, x_{0}\right)$. Since homeomorphisms need not fix $x_{0}$, this action is well defined only if we ignore the innerautomorphisms of $\pi_{1}\left(Q^{3}, x_{0}\right)$. More explicitly, if $h: Q^{3} \rightarrow Q^{3}$ is a homeomorphism, then $h$ is isotopic to $h_{1}$ where $h_{1}\left(x_{0}\right)=x_{0}$. Hence $h_{1}$ induces an automorphism $h_{1 *}$ of $\pi_{1}\left(Q^{3}, x_{0}\right)$. Let $h_{*}$ denote the element of Outaut $\pi_{1}\left(Q^{3}, x_{0}\right)=$ Aut $\pi_{1}\left(Q^{3}, x_{0}\right) /$ Inaut $\pi_{1}\left(Q^{3}, x_{0}\right)$ that contains $h_{1 *}$. Then the assignment $h \rightarrow h_{*}$ defines a homomorphism from $\mathscr{H}\left(Q^{3}\right)$ to the outerautomorphisms of $\pi_{1}\left(Q^{3}, x_{0}\right)$. 
The following two homeomorphisms correspond to nontrivial outerautomorphisms. Define $g_{1}: Q^{3} \rightarrow Q^{3}$ as follows. The image of $K^{2}$ is $F^{-1}(a) \times[0,1] \cup_{F} a$ together with an annulus, $A$, spanning $T^{3}$ (see Figure 2). The homeomorphism $g$, fixes $a$ (pointwise) and takes $b$ onto the oriented curve $l$.

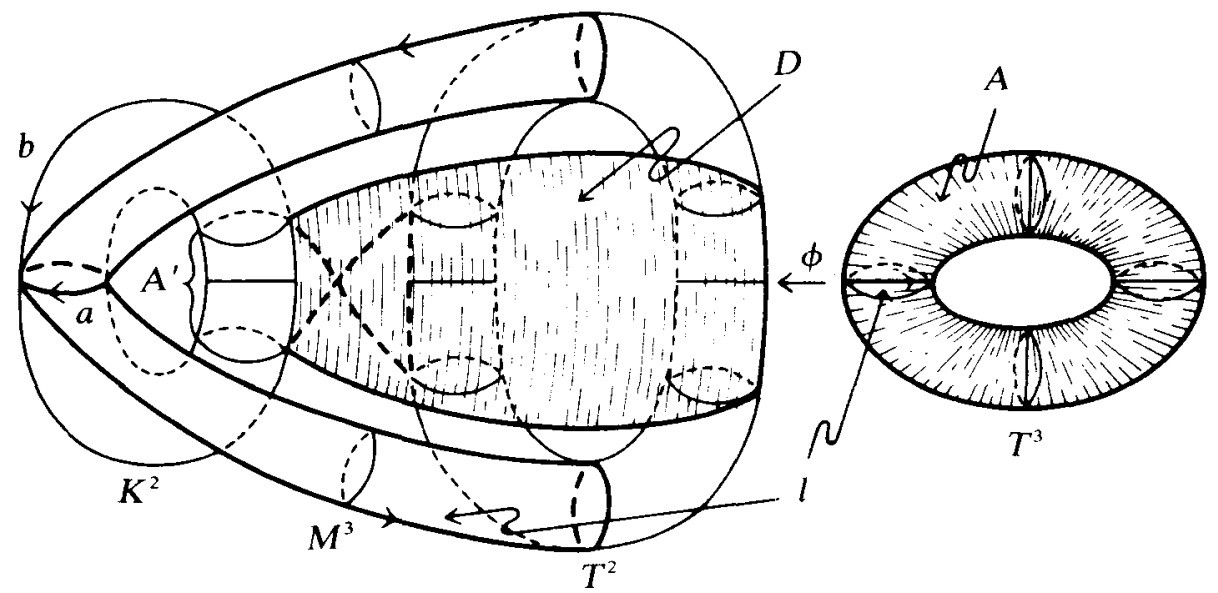

Figure 2

Note that in $\pi_{1}\left(Q^{3}, x_{0}\right),[l]=b a^{-1}=a b$. Hence the automorphism induced by $g_{1}$ takes $a$ to $a$ and $b$ to $a b$. So far we have described $g_{1}$ on $K^{2}$. Clearly $g_{1}$ extends to take $M^{3}$ onto closure $\left(Q^{3}-F^{-1}\left(A^{\prime}\right) \times[0,1] \cup F A^{\prime}\right)$. Since $F^{-1}\left(A^{\prime}\right) \times$ $[0,1] \cup_{F} A^{\prime}$ is a solid torus we need only check that the boundary of its meridional disk, $D$, sits properly on $\partial\left(g_{1}\left(M^{3}\right)\right)$. By sliding $\partial D$ towards $g_{i}\left(K^{2}\right)$ in Figure 2 one easily checks that $\partial D \sim$ lala $^{-1}$ so that $g_{1}$ extends to take $T^{3}$ onto $F^{-1}\left(A^{\prime}\right) \times[0,1] \cup_{F} A^{\prime}$. One final observation about $g_{1}$. Recall that $Q^{3}=\partial \nu$ where $\nu$ is the normal disk bundle of $P^{2}$ in $S^{4}$. In particular $Q^{3}$ is a circle bundle over $P^{2}$. The fibers of this bundle are just the meridians of $K^{2}$ and $T^{2}$ and the longitudes of $T^{3}$ (see Figure 1). It is easy to see that $g_{1}$ can be constructed to take fibers onto fibers (i.e. $g_{1}$ is a bundle map) and hence $g_{1}$ extends to a homeomorphism $G_{1}: \nu \rightarrow \nu$.

The next homeomorphism, $g_{2}$, is described similarly. The image of $K^{2}$ is $F^{-1}(b) \times[0,1] \cup_{F} b$ together with a Moebius band $M^{\prime}$ spanning $T^{3}$ (see Figure 3 ). The homeomorphism $g_{2}$ takes $a$ to $m$ and takes $b$ to $l$. 


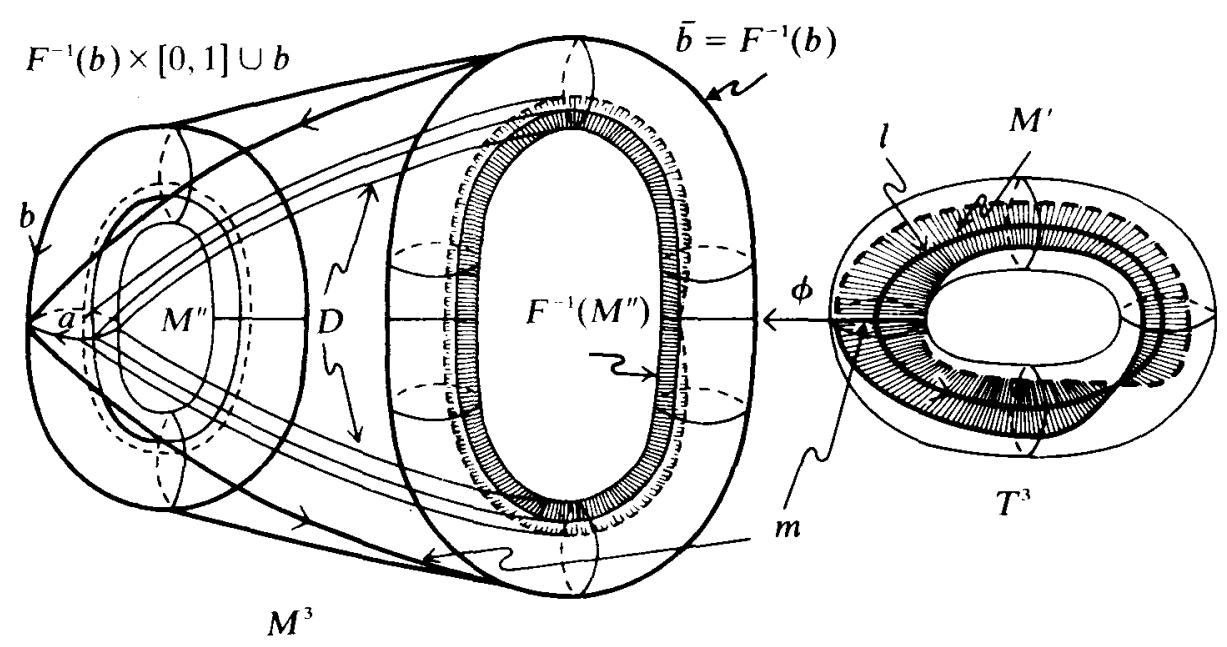

Figure 3

Note that the outerautomorphism induced by $g_{2}$ takes $a$ to $a b$ and takes $b$ to $a$. The above homeomorphism on $K^{2}$ extends to take $M^{3}$ onto closure $\left(Q^{3}-F^{-1}\left(M^{\prime \prime}\right) \times[0,1] \cup_{F} M^{\prime \prime}\right)$. Since $F^{-1}\left(M^{\prime \prime}\right) \times[0,1] \cup_{F} M^{\prime \prime}$ is a solid torus, we need only check that the boundary of its meridional disk, $D$, sits properly in $\partial\left(g_{2}\left(M^{3}\right)\right)$. By sliding $\partial D$ towards $g_{2}\left(K^{2}\right)$ in Figure 3 one easily checks that $\partial D=\operatorname{lm} / m^{-1}$ so that $g_{2}$ extends to take $T^{3}$ onto $F^{-1}\left(M^{\prime \prime}\right) \times[0,1] \cup_{F} M^{\prime \prime}$. Since kernel $\left(\pi_{1}\left(Q^{3}, x_{0}\right) \rightarrow \pi_{1}\left(\nu, x_{0}\right)\right)$ is $\left\{1, a, a^{2}, a^{-1}\right\}$ and since $g_{2 *}(a)=a b$ it follows that $g_{2}$ does not extend to $\nu$.

The next homeomorphism we need to construct is the most troublesome, but the easiest to describe. This homeomorphism, $g$, takes $K^{2}$ onto itself by taking $a$ to $a^{-1}$ and $b$ to $b^{-1}$. By Lemma 2.3 it extends to take $M^{3}$ onto $M^{3}$. Furthermore this extension takes $\partial D^{2} \subseteq \partial M^{3}$ onto itself (orientation reversing) so it extends to take $T^{3}$ onto $T^{3}$. The automorphism induced by $g$ takes $a$ to $a^{-1}$ and $b$ to $b^{-1}$. In particular the automorphism induced by $g$ is precisely conjugation by $a b$.

The above homeomorphisms are all orientation preserving homeomorphisms. For completeness we describe an orientation reversing homeomorphism, $r$. Let $\tau: T^{2} \rightarrow T^{2}$ be the nontrivial "covering translation" with $F \tau=F: T^{2} \rightarrow K^{2}$. Then $r$ is defined on $M^{3}$ by

$$
r(\theta,(x, y), t)=(\tau(\theta,(x, y)), t) .
$$

Note $r / K^{2}=$ identity. Since $r / T^{2}$ is just rotation half way around longitudinally it is isotopic to the identity and hence extends to take $T^{3}$ onto itself. 
Since $r$ is the identity on $K^{2}$ but, locally, switches points from one side of $K^{2}$ to the other it is orientation reversing. As with $g_{1}, r$ is fiber preserving and hence extends to $R: \nu \rightarrow \nu$.

\section{Isotopy classes of homeomorphisms of $Q^{3}$}

In this section we investigate the natural homomorphism of $\mathscr{I}\left(Q^{3}\right)=$ $\mathscr{H}\left(Q^{3}\right) / \mathscr{H}_{\text {id }}\left(Q^{3}\right)$ into Aut $\pi_{1}\left(Q^{3}, x_{0}\right) /$ Inaut $\pi_{i}\left(Q^{3}, x_{0}\right)$. Since $\pi_{1}\left(Q^{3}, x_{0}\right)$ is isomorphic to the quaternion group it is well known (see Passman (1968), page 74 for example) that Aut $\pi_{1}\left(Q^{3}, x_{0}\right)$ is isomorphic to $S_{4}(\equiv$ the symmetric group on 4 letters). The group of innerautomorphisms of $\pi_{1}\left(Q^{3}, x_{0}\right)$ is isomorphic to $\left\{a, b: a^{2}=b^{2}=(a b)^{2}=1\right\} \approx Z_{2} \oplus Z_{2}$. Hence the group of outerautomorphisms of $\pi_{1}\left(Q^{3}, x_{0}\right)$ is precisely $S_{3}$ (the symmetric group on 3 letters). The outerautomorphism $g_{1_{*}}$ has order $2\left(g_{1_{*}}^{2}(a)=a, g_{1_{*}}^{2}(b)=b^{-1}\right.$ which is just conjugation by $a$ ) while $g_{2 *}$ has order 3 . Hence $g_{1 *}$ and $g_{2 *}$ generate Outaut $\pi_{1}\left(Q^{3}, x_{0}\right)$.

Clearly any homeomorphism that is isotopic (in fact homotopic) to the identity induces an innerautomorphism. Hence $\mathscr{H}\left(Q^{3}\right) \rightarrow$ Outaut $\pi_{1}\left(Q^{3}, x_{0}\right)$ induces a homomorphism $\Psi: \mathscr{I}\left(Q^{3}\right) \rightarrow$ Outaut $\pi_{1}\left(Q^{3}, x_{01}\right)$. By the previous paragraph $\Psi$ is onto. The next few results show that $\Psi$ is $1-1$ also.

LeмMA 2.1. Let $J$ be a simple closed curve on $K^{2}$. Then either $J$ bounds a disk on $K^{2}$ or else $J$ is isotopic to one of $a, a b, b$ or $b^{2}$.

Proof. Suppose $J$ does not bound a disk. We assume $J$ has been isotoped so that it is transverse to $a$ and intersects $a$ as few times as possible. Now cut $K^{2}$ open along $a$ and we get an annulus, $A$. The curve $b$ becomes a spanning arc of $A$. If $J \cap a=\varnothing$, then $J$ is clearly isotopic to $a$. If $J \cap a \neq \varnothing$, then $J$ becomes a finite set of spanning arcs of $A$. If $J$ becomes one spanning arc, then $J$ is isotopic to either $b, a^{ \pm 1} b, a^{ \pm 2} b, \cdots$. Since $a^{2} b$ is homotopic to $b$, it follows from Theorem 3.3 of Epstein (1966) that $a^{n} b$ is isotopic to either $b$ or $a b$ depending on whether $n$ is even or odd. If $J$ becomes two spanning arcs, then $J$ is isotopic to $a^{n} b a^{n} b$ for some $n$. But $a^{n} b a^{n} b$ is isotopic to $b^{2}$. Finally it is easy to check that no simple closed curve $J$ will become more than two spanning arcs of $A$ when we cut along $a$.

LEMMA 2.2. Let $h: Q^{3} \rightarrow Q^{3}$ be a homeomorphism with $h_{*}$ being an innerautomorphism. Then $h$ is isotopic to $\bar{h}: Q^{3} \rightarrow Q^{3}$ with $\bar{h}\left(M^{3}\right)=M^{3}$.

Proof. Since any homeomorphism of a 3-manifold onto itself is isotopic to a $P L$ homeomorphism we can assume that $h$ is $P L$ and using general positioning we can assume that $h\left(K^{2}\right)$ intersects $K^{2}$ transversely in a finite 
collection of pairwise disjoint simple closed curves $J_{1}, J_{2}, \cdots, J_{n}$. Using the product structure on $M^{3}-K^{2}$ we can isotope $h$ (by pushing away from $K^{2}$ ) so that $M^{3}$ intersects the image of $K^{2}$ in a collection of pairwise disjoint annuli and Moebius bands $X_{1}, X_{2}, \cdots, X_{n}$ with $J_{i}$ being the centerline of $X_{i}$. For convenience of notation, we assume that $h$ has this property. We now isotope $h$ to simplify $K^{2} \cap h\left(K^{2}\right)$ so that we can push $h\left(T^{3}\right)$ onto $T^{3}$.

The first step is to eliminate all of the $J_{i}$ 's that are null homotopic on $K^{2}$. If such $J_{i}$ exists, then let $J$ be a minimal such curve (that is $J$ bounds a disk $D$ on $K^{2}$ with int $\left.D \cap h\left(K^{2}\right)=\varnothing\right)$. It follows that $J$ bounds a disk $D^{\prime}$ in $h\left(K^{2}\right)$ also because Lemma 2.1 lists all the isotopy classes of simple closed curves on $h\left(K^{2}\right)$ and of them only the null homotopic curve on $h\left(K^{2}\right)$ is null homotopic in $Q^{3}$. Thus $D \cup D^{\prime}$ is a 2-sphere and since $Q^{3}$ is irreducible $D \cup D^{\prime}$ bounds a 3-cell which must miss $h\left(K^{2}\right)-D^{\prime}$. By pushing $D^{\prime}$ across the 3-cell we can eliminate $J$ as a component of $K^{2} \cap h\left(K^{2}\right)$. In fact we assume we push $D^{\prime}$ so far that we eliminate the component of $M^{3} \cap h\left(K^{2}\right)$ containing $J$. Repeating this process we can eliminate all components of $K^{2} \cap h\left(K^{2}\right)$ that are null homotopic on $K^{2}$ (and because of the above argument this eliminates all components of $K^{2} \cap h\left(K^{2}\right)$ that are null homotopic on $h\left(K^{2}\right)$ also).

To simplify notation we assume that $h$ already had the above properties. That is we assume that $K^{2} \cap h\left(K^{2}\right)=J_{1} \cup \cdots \cup J_{m}$ and $M^{3} \cap h\left(K^{2}\right)=$ $X_{1} \cup \cdots \cup X_{m}$ where $J_{i}$ is a simple closed curve, $X_{i}$ is an annulus or a Moebius band with $J_{i}$ the centerline of $X_{i}$ and $J_{i}$ is not null homotopic on either $K^{2}$ or $h\left(K^{2}\right)$.

CASE 1: Some $J_{i}$ is isotopic, on $K^{2}$, to $a$.

Consider $J_{j}$ as a curve on $h\left(K^{2}\right)$. Since $h_{*}$ is an innerautomorphism we know that $h(a)$ is homotopic in $Q^{3}$, to $a^{ \pm 1}$. Hence $J_{j}$ is homotopic, in $Q^{3}$, to $a$ which is homotopic to $h(a)^{ \pm 1}$. Since none of $h(b), h(a b)$ or $h\left(b^{2}\right)$ are homotopic in $Q^{3}$ to $h(a)^{ \pm 1}$, it must be the case that $J_{j}$ is isotopic to $h(a)$ on $h\left(K^{2}\right)$. Thus the centerline of $h\left(T^{3}\right)$ is isotopic to $J_{i}$ which is isotopic to the centerline of $T^{3}$. It follows that $h$ is isotopic to $h_{1}$ with $h_{1}\left(T^{3}\right)=T^{3}$ and $h_{1}\left(M^{3}\right)=M^{3}$.

Case 2: No $J_{i}$ is isotopic, on $K^{2}$, to $a$.

In this case each $X_{i}$ is either a Moebius band with $J_{i}$ isotopic to $b$ or $a b$ or an annulus with $J_{i}$ isotopic to $b^{2}$. In either case each component of $\partial X_{i}$ is isotopic, on $\partial M^{3}$, to $\bar{b}$. Suppose $M$ is a component of $h\left(K^{2}\right)-\cup$ int $X_{i}$, with $M$ a Moebius band. Then the center line of $M$ must be isotopic, on $h\left(K^{2}\right)$, to $h(b)$ or $h(a b)$. Also, the centerline of $M$ must be isotopic in $T^{3}$ to $a$. Since $h_{*}$ is an innerautomorphism and since there is no innerautomorphism taking either $b$ or $a b$ to $a$ there can be no such $M$. Hence each component of 
$h\left(K^{2}\right)-\cup$ int $X_{i}$ is an annulus properly embedded in $T^{3}$ with boundary isotopic, on $T_{2}$, to $\bar{b}$. It is now easy to isotope $h$ to $h_{1}$ with $h_{1}\left(K^{2}\right) \cap T^{3}=\varnothing$. By squeezing $h_{1}\left(M^{3}\right)$ toward $h_{1}\left(K^{2}\right)$ we can assume further that $h_{1}\left(M^{3}\right) \cap T^{3}=\varnothing$ and hence that $T^{3} \subseteq h_{1}\left(T^{3}\right)$.

Finally we must show that $T^{3}$ and $h\left(T^{3}\right)$ are concentric. Let $A$ be the annulus starting at $\bar{a}$, following down the mapping cylinder structure of $M^{3}$ to $a$, then continuing on through the mapping cylinder structure to the other component of $F^{-1}(a) \subseteq \partial M^{3}$. We assume that $h_{1}\left(K^{2}\right)$ intersects $M$ in a finite collection of pairwise disjoint simple closed curves. Suppose one of these curves, call it $\Lambda$, is null homotopic in $A$. Then, as usual, we can assume it is minimal, that is $\Lambda$ bounds a disk $\Delta \subseteq A$ with int $\Delta \cap h_{1}\left(K^{2}\right)=\varnothing$. Then $\Lambda$ is null homotopic on $h_{1}\left(K^{2}\right)$ also because none of the curves $h_{1}(a), h_{1}(b), h_{1}(a b)$ or $h_{1}\left(b^{2}\right)$ is null homotopic in $M^{3}$. Thus $\Lambda$ bounds a disk $\Delta^{\prime} \subseteq h_{1}\left(K^{2}\right), \Delta \cup \Delta^{\prime}$ is a 2 -sphere and since $M^{3}$ is irreducible $h_{1}$ can be isotoped to eliminate $\Lambda$. Similarly we can eliminate all components of $A \cap h_{1}\left(K^{2}\right)$ that are null homotopic on $A$. To simplify notation we assume that $h_{1}$ had this property (that is we assume that no component of $A \cap h_{1}\left(K^{2}\right)$ is null homotopic on $A$ ). It follows that no component of $A \cap h_{1}\left(K^{2}\right)$ is null homotopic on $h_{1}\left(K^{2}\right)$ either, because it must be isotopic in $A$ to $a$ and hence can not be null homotopic in $Q^{3}$. Hence $A \cap h_{1}\left(K^{2}\right)$ is either empty or else consists of a family of pairwise disjoint simple closed curves all isotopic in $A$. If $A \cap h_{1}\left(K^{2}\right)$ were empty, then we would have $h_{1}\left(K^{2}\right) \subseteq M^{3}-A \simeq S^{1}$ which is impossible. Thus $A \cap h_{1}\left(K^{2}\right) \neq \varnothing$ and must consist of at least one simple closed curve. Let $\Lambda$ be a component of $A \cap h_{1}\left(K^{2}\right)$. Then $\Lambda$ is isotopic to $a$ on $A$ and $\Lambda$ is isotopic to $h_{1}(a), h_{1}(b), h_{1}(a b)$ or $h_{1}\left(b^{2}\right)$ on $h_{1}\left(K^{2}\right)$. Since $h_{1 *}$ is an innerautomorphism we must have $\Lambda$ isotopic to $h_{1}(a)$ on $h_{1}\left(K^{2}\right)$. Now, as in Case 1 , we have the centerline of $h_{1}\left(T^{3}\right)$ isotopic to $\Lambda$ which is isotopic to the centerline of $T^{3}$. Hence $h_{1}$ is isotopic to $h_{2}: Q^{3} \rightarrow Q^{3}$ with $h_{2}\left(T^{3}\right)=T^{3}$ and $h_{2}\left(M^{3}\right)=M^{3}$.

LEMMA 2.3. Let $h: \dot{K}^{2}, x_{0} \rightarrow K^{2}, x_{0}$ be a homeomorphism. If $h_{*}\left(F_{*}\left(\pi_{1}\left(T^{2}, e_{0}\right)\right)\right) \subseteq F_{*}\left(\pi_{1}\left(T^{2}, e_{0}\right)\right)$, then $h$ extends to a level preserving homeomorphism $\bar{h}: M^{3} \rightarrow M^{3}$. Furthermore if $H: K^{2} \times I \rightarrow K^{2} \times I$ is an isotopy with $H(x, 0)=h(x)$, then $H$ extends to an isotopy $\bar{H}: M^{3} \times I \rightarrow M^{3} \times I$ with $\bar{H}(x, 0)=\bar{h}$.

Proof.

$$
\begin{array}{cr}
T^{2}, e_{0} & T^{2}, e_{0} \\
\downarrow F & \downarrow F \\
K^{2}, x_{0} \rightarrow & K^{2}, x_{0} .
\end{array}
$$

Since $h_{*} F_{*}\left(\pi_{1}\left(T^{2}, e_{0}\right)\right) \subseteq F_{*}\left(\pi_{1}\left(T^{2}, e_{0}\right)\right)$ it follows that $h_{*} F_{*}$ lifts to $h^{\prime}:\left(T^{2}, e_{0}\right) \rightarrow\left(T^{2}, e_{0}\right)$. Define $\bar{h}: M^{3} \rightarrow M^{3}$. 


$$
\begin{aligned}
& \bar{h}(z, t)=\left(h^{\prime}(z), t\right) \text { for } \quad(z, t) \in T^{2} \times I \\
& \bar{h}(x)=h(x) \quad \text { for } \quad x \in K^{2}
\end{aligned}
$$

The extension of the isotopy is obtained the same way.

$$
\begin{array}{cc}
T^{2} \times I, e_{0} \times 0 & T^{2} \times I, e_{0} \times 0 \\
\downarrow F \times \mathrm{id} & \downarrow F \times \mathrm{id} \\
K^{2} \times I, x_{0} \times 0 \rightrightarrows & K^{2} \times I, x_{0} \times 0
\end{array}
$$

$H \circ(F \times$ id $)$ lifts to $H^{\prime}$. Then define

$$
\begin{array}{ll}
\bar{H}((z, t) \times s)=\left(H^{\prime}(z, t), s\right) & \text { for } \quad(z, t) \times s \in\left(T^{2} \times I\right) \times I \\
\bar{H}(x \times s)=H(x \times s) & \text { for } \quad x \times s \in K^{2} \times I .
\end{array}
$$

Lemma 2.4. Let $h: M^{3} \rightarrow M^{3}$ be a homeomorphism. The $h$ is ambient isotopic to $h_{1}: M^{3} \rightarrow M^{3}$ with $h_{1}$ level preserving. In particular $h_{1}\left(K^{2}\right)=K^{2}$.

Proof. We assume that $h$ is $P L$, then the result follows easily from Lemma 2.3 and Theorem 7.1 of Waldhausen (1968). Consider the following composition of maps $K^{2} \stackrel{s}{\rightarrow} M^{3} \stackrel{h}{\rightarrow} M^{3} \stackrel{\text { proj }}{\rightarrow} K^{2}$. Since this composition is a homotopy equivalence it induces a homeomorphism $h_{1}: K^{2} \rightarrow K^{2}$. Since $h$ sends $T^{2}=\partial M^{3}$ to itself it follows that the above homotopy equivalence sends $\left(F_{*}\left(\pi_{1}\left(T^{2}\right)\right)\right.$ onto itself and hence by Lemma $2.3 h_{1}$ extends to a level preserving homeomorphism $h_{1}: M^{3} \rightarrow M^{3}$. That $h$ is isotopic to $h_{1}$ follows from Waldhausen's result since $h$ is clearly homotopic to $h_{1}$.

The idea for the proof of the next lemma and more importantly the impetus for believing it was true is due to $\mathrm{J}$. H. Rubinstein. It is a nice addition to the original manuscript since it provides a complete computation of $\mathscr{I}\left(Q^{3}\right)$. I am grateful for his help on this lemma.

Lemma 2.5. The homeomorphism $\mathrm{g}$, described in the previous section, is isotopic to the identity.

Proof. We must first define some sets to keep track of during the isotopy. Let $N_{a b}, N_{a}$ and $N_{b}$ be 3-cells with $x_{0}=a \cap b \subseteq$ int $N_{a b}, N_{a b} \cup N_{a}$ is a regular neighborhood of $a, N_{a b} \cup N_{b}$ is a regular neighborhood of $b$ and $\left(N_{a b} \cup N_{a}\right) \cap\left(N_{a b} \cup N_{b}\right)=N_{a b}$. Furthermore, we assume that (for $i=a, b, a b$ ) we have $N_{i} \cap K^{2}=D_{i}$ is a 2-disk on $K^{2}$ with $D_{a b} \cup D_{a}$ a regular neighborhood of $a$ in $K^{2}$ and $D_{a b} \cup D_{b}$ a regular neighborhood of $b$ in $K^{2}$.

The homeomorphism $g_{2}^{-1} g_{1}$ takes $K^{2}$ to a Klein bottle that intersects $K^{2}$ in $b$ and a meridian. Furthermore $b$ is a meridian on $g_{2}^{-1} g_{1}\left(K^{2}\right)$ and the meridian on $K^{2}$ is a longitude on $g_{2}^{-1} g_{1}\left(K^{2}\right)$. By sliding $g_{2}^{-1} g_{1}\left(K^{2}\right)$ around we can get a Klein bottle, $\bar{K}^{2}$, that intersects $K^{2}$ in $a \cup b$ and $a$ is a longitude on 
$\bar{K}^{2}$ while $b$ is a meridian on $\bar{K}^{2}$. For convenience we adjust $\bar{K}^{2}$ slightly so that $\bar{K}^{2} \cap K^{2}=D_{a b} \cup a \cup b$. Finally we assume that $\bar{D}_{i}=N_{i} \cap \bar{K}^{2}$ is a disk (of course $\bar{D}_{a b}=D_{a b}$ ).

We can now start isotoping $g$ to the identity. Let $h_{1}: Q^{3} \rightarrow Q^{3}$ be the homeomorphism obtained by sliding $a$ around $K^{2}$, keeping $b$ setwise fixed, to $a^{-1}$. (Define $h_{1}$ first on $K^{2}$, then extend to $M^{3}$ using Lemma 2.3 and finally extend to $T^{3}$ using the fact that the part already defined is isotopic to the identity.) Then $h_{1}$ is clearly isotopic to the identity, hence $g$ is isotopic to $h_{1} g$. Furthermore we can assume that (for $i=a, b, a b) h_{1} g\left(D_{i}\right)=D_{i}$. Note that $h_{1} g(a)=a$ while $h_{1} g(b)=b^{-1}$. Next we slide $b$ around $\bar{K}^{2}$. More precisely, let $h_{2}: Q^{3} \rightarrow Q^{3}$ be the homeomorphism obtained by sliding $b$ around $\bar{K}^{2}$, keeping $a$ setwise fixed, to $b^{-1}$. As with $h_{1}, h_{2}$ is isotopic to the identity, so $g$ is isotopic to $h_{2} h_{1} g$. Furthermore we can assume that (for $\left.i=a, b, a b\right) h_{2}\left(\bar{D}_{i}\right)=$ $\left.\bar{D}_{i}\right)$. Note that $h_{2} h_{1} g(a)=a, h_{2} h_{1} g(b)=b$ and $h_{2} h_{1} g\left(D_{a b}\right)=D_{a b}=\bar{D}_{a b}$. By regular neighborhood theory we can assume that (for $i=a, b, a b$ ) $h_{2} h_{1} g\left(N_{i}\right)=N_{i}$. We can also assume that $h_{2} h_{1} g \mid D_{a b}$ is the identity. It is not very clear what happens to $D_{i}=h_{1} g\left(D_{i}\right)(i=a, b)$ under $h_{2}$. But Figure 4 shows that we can in fact assume that $h_{2}\left(h_{1} g\left(D_{b}\right)\right)=h_{2}\left(D_{b}\right)=D_{b}$ also (because $h_{2}$ takes $N_{b}$ orientation preserving onto $N_{b}$ but takes $\bar{D}_{b}, b$ orientation reversing onto $\left.\bar{D}_{b}, b\right)$. Similarly we can assume that $h_{2}\left(h_{1} g\left(D_{a}\right)\right)=$

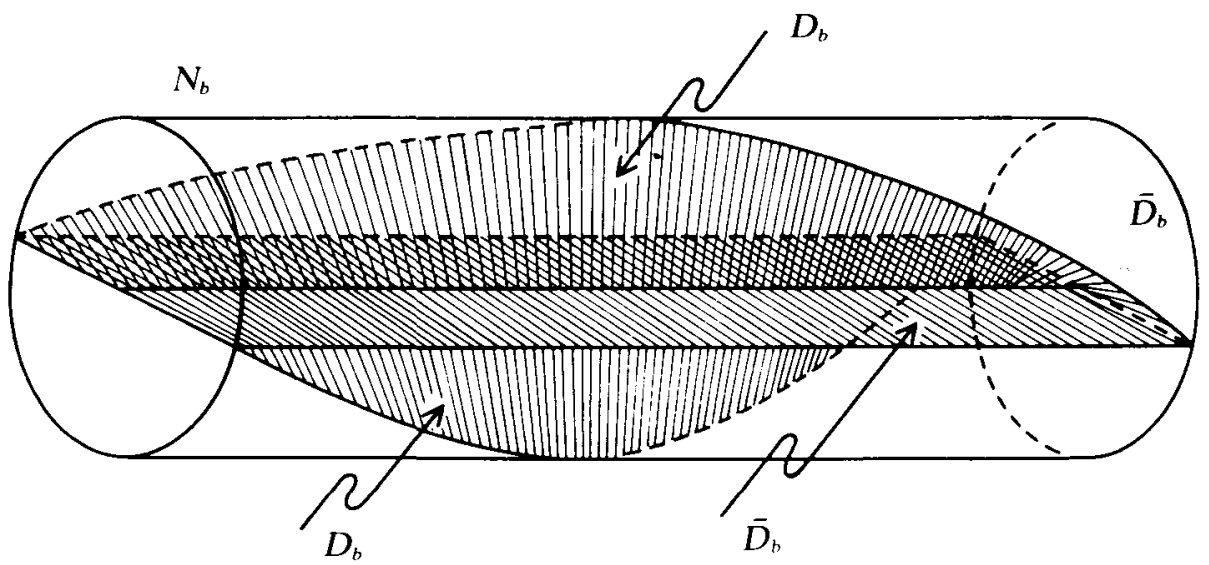

Figure 4

$D_{a}$. In fact since $h_{2} h_{1} g$ takes $a$ to $a$ and $b$ to $b$, we can assume that $h_{2} h_{1} g \mid D_{a} \cup D_{b} \cup D_{a b}$ is the identity. The remainder of the proof is straightforward. Let $D$ be the disk closure $\left(K^{2}-\left(D_{a} \cup D_{b} \cup D_{a b}\right)\right)$. Then $\partial D=h_{2} h_{1} g(\partial D)$. We can adjust $h_{2} h_{1} g$ so that $h_{2} h_{1} g(D)$ is in general position 
with respect to $D$, relative to $\partial D$. Hence $h_{2} h_{1} g(D) \cap D$ is a collection of pairwise disjoint simple closed curves in $D$. Since.they lie in $D$ they are null homotopic on $K^{2}$ and hence can be eliminated as in the proof of Lemma 2.2. Eventually this process isotopes $h_{2} h_{1} g$ to a homeomorphism $h_{3}: Q^{3} \rightarrow Q^{3}$ with $h^{3} \mid D_{a} \cup D_{b} \cup D_{a b}$ the identity and $h_{3}(D) \cap D=\partial D=h_{3}(\partial D)$. By irreducibility $D \cup h_{3}(D)$ bounds a 3 -cell and we can push $h_{3}(D)$ across the 3 -cell to $D$. In other words $h_{3}$ is isotopic to $h_{4}: Q^{3} \rightarrow Q^{3}$ with $h_{4}(a)=a$, $h_{4}(b)=b$ and $h_{4}\left(K^{2}\right)=K^{2}$. It is easy to see that $h_{4}$ is isotopic to the identity (see Case I of the proof of Theorem 1.)

THEOREM 1. Let $h: Q^{3} \rightarrow Q^{3}$ be an orientation preserving homeomorphism. If $h_{*}$ is an innerautomorphism, then $h$ is isotopic to the identity.

Proof. By Lemma 2.2, $h$ is isotopic $h_{1}: Q^{3} \rightarrow Q^{3}$ with $h_{1}\left(M^{3}\right)=M^{3}$. By Lermma $2.4, h_{1} \mid M^{3}$ is isotopic to $h_{2}: M^{3} \rightarrow M^{3}$ with $h_{2}\left(K^{2}\right)=K^{2}$. Using a collar neighborhood of $\partial M^{3}=\partial T^{3}$ in $T^{3}$ we can extend $h_{2}$ to all of $M^{3}$ and get $h_{1}$ is isotopic to $h_{2}: Q^{3} \rightarrow Q^{3}$ with $h_{2}\left(K^{2}\right)=K^{2}$. Since $h_{*}$ is an inner automorphism so is $h_{2 *}$. Hence $h_{2}(a)$ is a simple closed curve on $K^{2}$ and it must be conjugate as a loop in $\pi_{1}\left(Q^{3}, x_{0}\right)$ to $a$. Thus $h_{2}(a)$ is isotopic on $K^{2}$ to $a^{ \pm 1}$. Similarly $h_{2}(b)$ is isotopic to $b^{ \pm 1}$.

CASE I: $h_{2}(a)$ is isotopic, on $K^{2}$, to $a^{ \pm 1}$ and $h_{2}(b)$ is isotopic to $b$.

Since there is an ambient isotopy taking $a$ to $a^{-1}, b$ to $b$ and $K^{2}$ onto $K^{2}$ we can assume that $h_{2}(a)=a$ and $h_{2}(b)=b$. Clearly $h_{2} \mid K^{2}$ is isotopic to the identity so by Theorem 7.1 of Waldhausen (1968) $h_{2} \mid M^{3}$ is isotopic to the identity. Using a collar neighborhood of $\partial M^{3}=\partial T^{3}$ in $T^{3}$ the isotopy extends to all of $Q^{3}$. Hence $h_{2}$ is isotopic to $h_{3} ; Q^{3} \rightarrow Q^{3}$ with $h_{3} \mid M^{3}=$ identity. Since $h_{3} \mid T^{3}: T^{3} \rightarrow T^{3}$ is the identity on the boundary, it is isotopic to the identity keeping the boundary fixed. Extending this isotopy, to be the identity on $M^{3}$, we have the desired result in this case.

CASE II: $h_{2}(a)$ is isotopic, on $K^{2}$, to $a^{ \pm 1}$ and $h_{2}(b)$ is isotopic, on $K^{2}$, to $b^{-1}$.

Since there is an ambient isotopy taking $a$ to $a^{-1}, b$ to $b$ and $K^{2}$ to $K^{2}$ we can assume that $h_{2}(a)=a^{-1}$ and $h_{2}(b)=b^{-1}$. Then $h_{2} \mid K^{2}$ is clearly isotopic to $g \mid K^{2}$. Appealing to Waldhausen's result again it follows, as in Case I, that $h_{2}$ is isotopic to $\mathrm{g}$. By Lemma $2.5 \mathrm{~g}$ is isotopic to the identity and the result follows.

Corollary. The sequence $0 \rightarrow \mathscr{H}_{\mathrm{id}}\left(Q^{3}\right) \stackrel{\subseteq}{\hookrightarrow} \mathscr{H}\left(Q^{3}\right) \rightarrow$ Outaut $\pi_{1}\left(Q^{3}, x_{0}\right) \rightarrow 0$ is exact. In particular $\mathscr{H}\left(Q^{3}\right) / \mathscr{H}_{\mathrm{id}}\left(Q^{3}\right)=\mathscr{I}\left(Q^{3}\right) \approx S_{3}$ (symmetry group on three letters). 
Corollary. Let $h: Q^{3} \rightarrow Q^{3}$ be a homeomorphism. Then $h$ is homotopic to the identity iff $h$ is isotopic to the identity.

\section{Projective planes in $S^{4}$}

In this section we use the characterization of homeomorphisms of $Q^{3}$ to study embeddings of the projective plane $P^{2}$ in $S^{4}$. We use the following notation. The symbol $\nu$ always refers to the normal disk bundle of the standard projective plane in $S^{4}$. As we mentioned earlier, the normal disk bundle of any $P L$ locally flat projective plane in $S^{4}$ is $P L$ homeomorphic to $\nu$. Recall that $Q^{3}=\partial \nu$ with the fibers (as a 1-sphere bundle over $P^{2}$ ) being the meridians of $K^{2}, T^{2}$, etc. and the longitudes of $T^{3}$. The symbols $a$ and $b$ will be used. as before, to denote elements of $\pi_{1}\left(Q^{3}, x_{0}\right)$ and to denote simple closed curves in $Q^{3}$. We always assume that $a$ is a meridian of $K^{2}$, in particular $a$ generates the kernel of $\pi_{1}\left(Q^{3}, x_{0}\right) \subseteq \pi_{1}\left(\nu, x_{0}\right)$. If $P$ is any $P L$ locally flat projective plane in $S^{4}$ with normal disk bundle $\nu(P)$, then we identify $\nu(P)$ with $\nu$ and hence $\partial \nu(P)$ with $Q^{3}$ by a homeomorphism. Of course the image of a generates kernel $\pi_{1}(\partial \nu(P), x) \stackrel{\complement}{\rightarrow} \pi_{1}(\nu(P), x)$.

The symbols $g_{1}$ and $g_{2}$ will be used to denote the homeomorphisms described in Section I. We use $G_{1}$ to denote the extension to all of $\nu$ of $g_{1}$. By the results of Section II, each isotopy class of homeomorphisms induces a unique permutation of the symbols $a, b, a b$. The following table lists some representatives of each class.

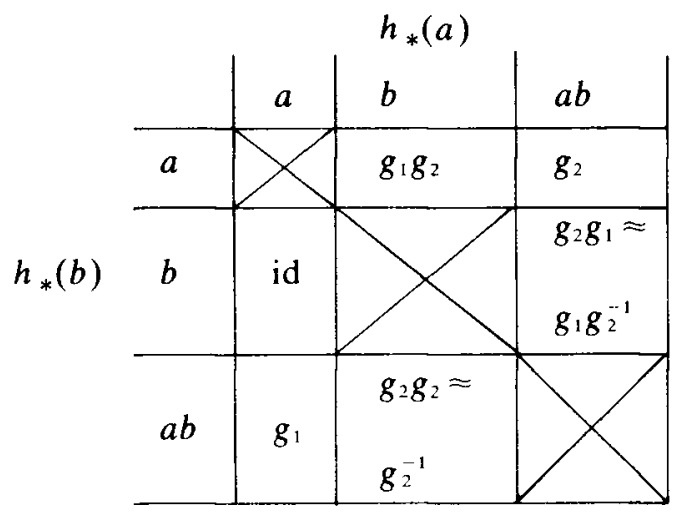

THEOREM 2. Let $H: \nu \rightarrow \nu$ be a homeomorphism. Then $h=H \mid Q^{3}$ is isotopic to the identity or $\mathrm{g}_{1}$.

Proof. Since $a$ generates the kernel $\pi_{1}\left(Q^{3}, x_{0}\right) \stackrel{c}{\rightarrow} \pi_{1}\left(\nu, x_{0}\right)$ it follows that $h_{*}(a)=a$. The result now follows from the above table of isotopy classes. 
THEOREM 3. There exist at most two distinct locally flat embeddings of $P^{2}$ in $S^{4}$ with a given closed complement.

Proof. Let $f_{1}, f_{2}: P^{2} \rightarrow S^{4}$ be locally flat embeddings. Suppose $\nu_{1}$ and $\nu_{2}$ are normal disk bundles of $f_{1}\left(P^{2}\right)$ and $f_{2}\left(P^{2}\right)$ respectively. Let $X_{i}=S^{4}-$ int $\nu_{i}$. Then there exist homeomorphisms $\phi_{i}:\left(P^{2}, \nu\right) \rightarrow\left(f_{i}\left(P^{2}\right), \nu_{i}\right)$ and $\left(f_{i}\left(P^{2}\right), S^{4}\right)$ is homeomorphic to $\left(P^{2}, v \cup_{\phi_{i}} X_{i}\right)$. Furthermore we can assume that $\phi_{i}(b)$ generates kernel $H_{1}\left(\partial \nu_{i} ; Z\right) \rightarrow H_{1}\left(S^{4}-f_{i}\left(P^{2}\right) ; Z\right)$. (Either $\phi_{i}(b)$ or $\phi_{i}(a b)$ generates it. If $\phi_{i}(a b)$ generates it then replace $\phi_{i}$ by $\phi_{i} G_{1}:\left(P^{2}, \nu\right) \rightarrow\left(f_{i}\left(P^{2}\right), \nu_{i}\right)$. $)$

Now suppose $H: X_{1} \rightarrow X_{2}$ is a homeomorphism. Then $\left.\phi_{2}^{-1} H \phi_{1}\right|_{Q^{7}}: Q^{3} \rightarrow Q^{3}$ and $\phi_{2}^{-1} H \phi_{1 *}(b)=b$. Hence $\phi_{2}^{-1} H \phi_{1}$ is either isotopic to the identity in which case $\left(f_{1}\left(P^{2}\right), S^{4}\right)$ is homeomorphic to $\left(f^{2}\left(P^{2}\right), S^{4}\right)$ or else $\left.\phi_{2}^{-1} H \phi_{1}\right|_{Q^{3}}$ is isotopic to $g_{2} g_{1}$. In this latter case all we can say is that $\left(f_{1}\left(P^{2}\right), S^{4}\right)$ is homeomorphic to $\left(f_{2}\left(P^{2}\right), S^{4}\right)$ iff there exists a homeomorphism $H_{1}: X_{1} \rightarrow X_{1}$ with $H_{1 *}(b)=b$ and $H_{1 *}(a)=a b$. In any case there are at most two embeddings whose closed complement is homeomorphic to $X_{1}$.

Corollary. Let $P$ be a locally flat projective plane in $S^{4}$. If the closed complement of $P$ is homeomorphic to the closed complement of the standard embedding, $P^{2}$, then $\left(P, S^{4}\right)$ is homeomorphic to $\left(P^{2}, S^{4}\right)$.

Proof. Figure 5 shows two linking projective planes whose normal disk bundles fill up $S^{4}$. Since the upper one is the standard embedding $P^{2}$, it follows that its closed complement is homeomorphic to $\nu$ (see Price and Roseman (1975) for a more detailed discussion). By the remark at the end of the last proof it is sufficient to exhibit a homeomorphism of the closed complement, $\approx v$, onto itself that fixes the curve generating the kernel $H_{1}(\partial \nu ; Z) \rightarrow H_{1}\left(S^{4}-P ; Z\right)$ while interchanging the other curves. Since the rolls of $a$ and $b$ have been reversed here this is exactly what the homeomorphism $G_{1}: \nu \rightarrow \nu$ does.

In Price and Roseman (1975) the notions of spun and twist spun projective planes were developed. Theorem 5.4 of that paper states: If $P_{0}$ and $P_{1}$ are spun projective planes constructed from $P^{2}$ by sewing $\left(N_{h}, M_{h}\right)$ onto $S^{4}-S^{1} \times B^{2} \times[-1,1]$ with $k_{1}$ and $k_{1}$ twists respectively then $S^{4}-P_{0}$ is homeomorphic to $S^{4}-P_{1}$ if $k_{0} \equiv k_{1} \bmod 2$. While it is not worthwhile giving all the definitions necessary to give a rigorous proof here it is easy to check that the homeomorphism constructed there was actually defined on the closed complements and it takes $a_{0}$ to $a_{1}\left(a_{i}\right.$ denotes the generator of kernel $\left.\pi_{1}\left(\partial \nu\left(P_{i}\right)\right) \rightarrow \pi_{1}\left(\nu\left(P_{i}\right)\right)\right)$. Hence that homeomorphism extends to take $\left(P_{0}, S^{4}\right)$ onto $\left(P_{1}, S^{4}\right)$. Thus we have the following. 


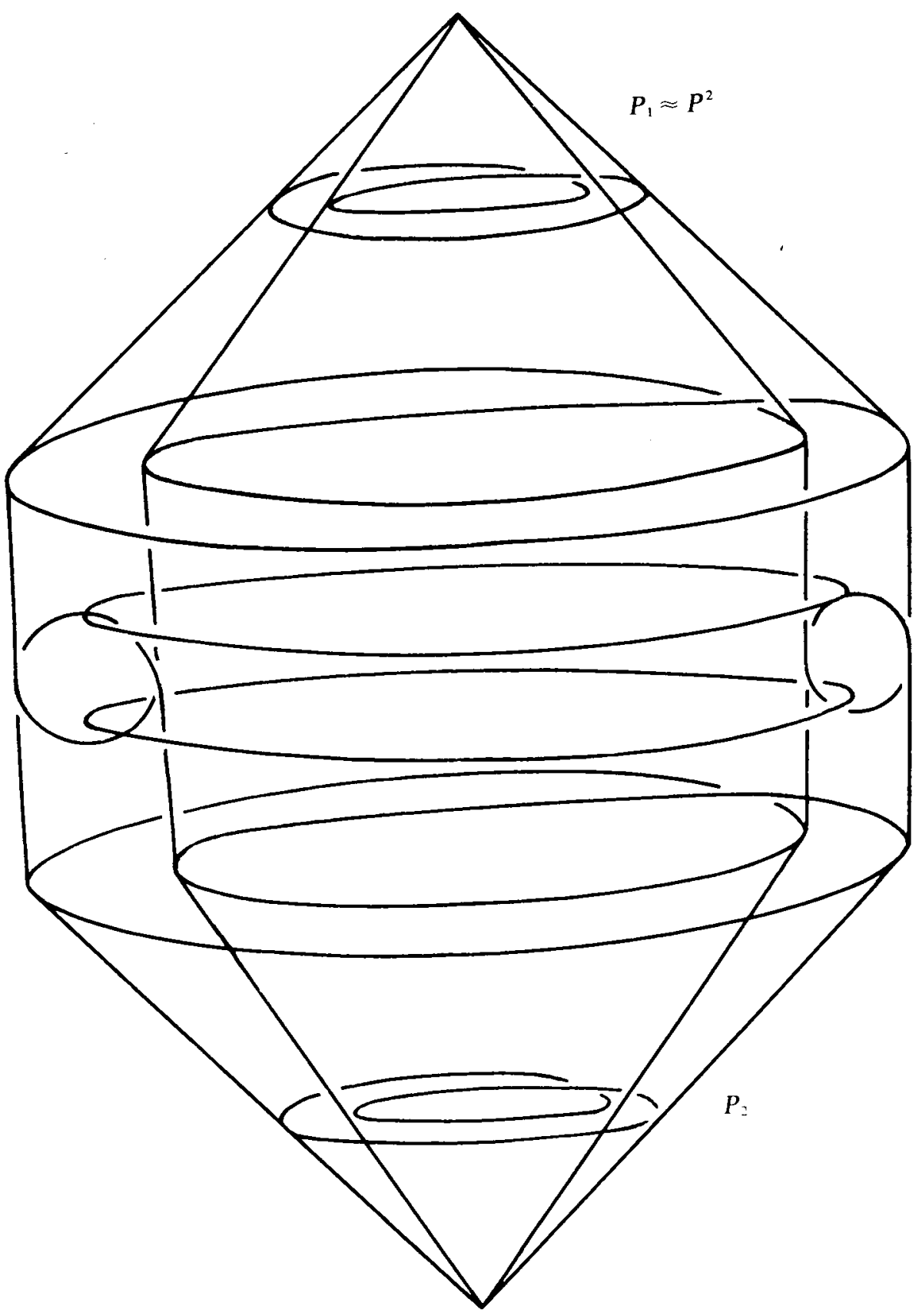

Figure 5 
Corollary. Let $P_{0}$ and $P_{1}$ be twist spun projective planes with $k_{0}$ and $k_{1}$ twists respectively (as in the discussion above). If $k_{0} \equiv k_{1} \bmod 2$, then $\left(P_{0}, S^{4}\right)$ is homeomorphic to $\left(P_{1}, S^{4}\right)$.

THEOREM 4. There are exactly two 4-manifolds that can be constructed by sewing $\nu$ to itself by some homeomorphism of $\partial \nu=Q^{3}$ onto itself. One being $S^{4}$, the other having fundamental group $Z_{2}$.

Proof. Let $h: Q^{3} \rightarrow Q^{3}$ be a homeomorphism. Clearly $\nu \cup_{h} \nu$ depends only on the isotopy class of $h$, hence (counting orientation reversing homeomorphisms) there are at most twelve such manifolds. If $h: Q^{3} \rightarrow Q^{3}$ is orientation reversing then $\nu \cup_{h} \nu$ is homeomorphic (see diagram below) to $\nu \cup_{r h} \nu$ and $r h$ is orientation preserving. Hence there are at most six such manifolds.

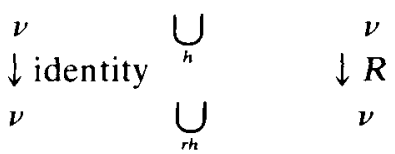

Similarly the manifold $W=\nu \cup_{\text {id }} \nu$ is homeomorphic to $\nu \cup_{\mathrm{g} 1} \nu$ because of the following diagram.

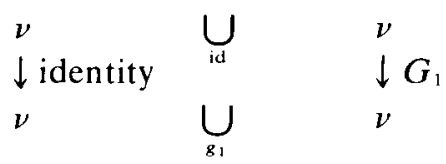

Also Van Kampen's theorem gives $\pi_{1}\left(W, x_{0}\right) \approx Z_{2}$. The Mayer-Vietoris sequence yields $H_{2}(W, Z) \approx Z_{2}, H_{3}(W, Z) \approx 0$ and $H_{4}(W, Z) \approx Z$. Using the same type of homeomorphism as above $\nu \cup_{g_{182}} \nu$ is homeomorphic to $\nu \cup_{g_{2}} \nu$ and similarly $\nu \cup_{g 12^{-1}} \nu$ is homeomorphic to $\nu \cup_{g^{-1}} \nu$. Finally by taking the first term of $\nu \cup_{g_{2}} \nu$ "identically" onto the second term of $\nu \cup_{z_{2}^{-1}} \nu$ and the second term of $\nu \cup_{g_{2}} \nu$ "identically" onto the first term of $\nu \cup_{g_{2}^{-1}} \nu$ we see that $\nu \cup_{g_{2}} \nu$ is homeomorphic to $\nu \cup_{g z^{\prime}} \nu$. Since it is easy to construct two linking projective planes in $S^{4}$ whose normal disk bundles fill up $S^{4}$ (see Figure 5) (see Price and Roseman (1975) for a more complete discussion) there must be some way of sewing $\nu$ to $\nu$ to get $S^{4}$. Since $W$. is not $S^{4}$ and since the other four spaces are all homeomorphic, they must all be homeomorphic to $S^{4}$.

\section{References}

A. Borel (1953), 'La cohomologie $\bmod 2$ de certains espaces homogènes', Comm. Math. Helv. 27, $165-197$.

D. B. A. Epstein (1966), 'Curves on 2-manifolds and isotopies', Acta. Math. 115, 83-107. 
H. Gluck (1962), 'The embeddding of two-spheres in the four sphere', Trans. Amer. Math. Soc. 104, 308-333.

W. S. Massey (1969), 'Proof of a conjecture of Whitney', Pacific J. Math. 31, 143-156.

W. S. Massey (1974), 'Imbeddings of projective planes and related manifolds in spheres', Ind. Math. J. 23, 791-812.

D. S. Passman (1968), Permutation Groups, W. A. Benjamin.

T. M. Price and D. M. Roseman (1975), 'Embeddings of the projective plane in four space', submitted.

H. Seifert and W. Threlfall (1934), Lehrbuch der Topologie, Leipzig: Teubner.

F. Waldhauser (1968), 'On irreducible 3-manifolds which are sufficiently large', Ann. of Math. 87, $56-88$.

Department of Mathematics,

University of Melbourne,

Victoria 3052, Australia

and

University of Iowa,

Iowa City,

Iowa 52242,

U.S.A. 